\title{
Perfil epidemiológico de 58 portadores de síndrome de Klippel-Trénaunay-Weber acompanhados no Ambulatório da Santa Casa de São Paulo
}

\author{
Epidemiological profile of 58 patients with Klippel-Trenaunay-Weber \\ syndrome followed at Ambulatório da Santa Casa de São Paulo
}

\begin{abstract}
André Luis Costa Villela, Luis Gustavo Schaefer Guedes, Victor Vinicius Augusto Paschoa, André Bernardes David, Thiago Marques Tenório, Henrique Pereira Lamego Junior, Henrique Jorge Guedes Neto, Roberto Augusto Caffaro*
\end{abstract}

\begin{abstract}
Resumo
Contexto: A síndrome de Klippel-Trénaunay-Weber é uma doença rara sobre a qual encontramos poucos artigos na literatura (geralmente relatos de casos esporádicos relacionados a complicações).

Objetivo: Avaliar o perfil epidemiológico dos portadores da referida síndrome.

Métodos: Foram copilados dados dos prontuários de 58 pacientes acompanhados no ambulatório de doenças linfáticas e angiodisplasias da disciplina de Cirurgia Vascular da Faculdade de Ciências Médicas da Santa Casa de São Paulo.
\end{abstract}

Resultados: A distribuição foi igual entre homens e mulheres (30 homens e 28 mulheres). A idade média dos pacientes em tratamento foi de 12,8 anos. Na maioria dos casos, a doença foi diagnosticada na infância, sendo a mancha em vinho do porto o primeiro sinal notado pela família, no nascimento ou primeiro ano de vida. O sintoma mais referido foi a dor, normalmente relacionada aos sintomas de estase venosa, sendo o sintoma considerado debilitante. Pela classificação CEAP, encontramos as crianças nas classes $\mathrm{C} 0$ e $\mathrm{C} 1$ e a maioria dos adultos nas classes avançadas. Foi diagnosticada a presença de fístulas arteriovenosas em $8,5 \%$ dos casos. Apenas 6,8\% referiram história familiar.

Conclusão: A síndrome de Klippel-Trénaunay e a síndrome de Parkes Weber são apresentações diferentes de uma única enfermidade e podem ser estudadas conjuntamente como síndrome de Klippel-Trénaunay-Weber. O melhor momento para reconhecer os portadores e poder amenizar a progressão de insuficiência venosa, hipertrofia óssea e tecidos moles é a infância. $\mathrm{O}$ tratamento compressivo deve ser indicado a todos os portadores com o intuito de diminuir a evolução da doença venosa periférica.

Palavras-chave: Angiodisplasias, síndrome de Klippel-Trénaunay-Weber, vascular, síndrome.

\section{Introdução}

Síndrome de Klippel-Treénaunay-Weber (SKTW) é o nome pelo qual é conhecido um conjunto de sinais que consiste em mancha em vinho do porto, anomalias venosas

\begin{abstract}
Background: Klippel-Trenaunay-Weber syndrome is a rare disorder about which there are few articles available (most of them are sporadic case reports related to complications).

Objective: To assess the epidemiological profile of patients with Klippel-Trenaunay-Weber syndrome.

Methods: Medical records of 58 patients followed at the lymphatic disease and angiodysplasia outpatient clinic for the discipline of Vascular Surgery at the Faculdade de Ciências Médicas da Santa Casa de São Paulo were reviewed.

Results: The disorder affected males and females equally ( 30 males and 28 females). The mean age of patients under treatment was 12.8 years. In most cases, the syndrome had been diagnosed in childhood, with Port wine stain as the first sign noticed by the family at birth or during the first year of life. The most frequently reported symptom was debilitating pain usually associated with symptoms of venous stasis. The CEAP classification was $\mathrm{C} 0$ and $\mathrm{C} 1$ for younger patients and $\mathrm{C} 4-\mathrm{C} 6$ for most older patients. Arteriovenous fistulas were diagnosed in $8.5 \%$ of cases. Positive family history was reported by only $6.8 \%$ of patients.
\end{abstract}

Conclusion: Klippel-Trenaunay and Parkes Weber syndromes are different presentations of a same disorder and they can be studied as one, Klippel-Trenaunay-Weber syndrome. Childhood is the optimal moment to identify affected patients and to reduce progression of venous insufficiency and bony and soft tissue hypertrophy. Compression should be indicated for all affected patients to reduce progression of peripheral venous disease.

Keywords: Angiodysplasias, Klippel-Trenaunay-Weber syndrome, vascular, syndrome.

e hipertrofia de ossos e tecidos moles, ou, pelo menos, dois desses sinais clínicos.

Foi descrita inicialmente em 1900 por Maurice Klippel e Paul Trénaunay, que relataram dois casos que

\footnotetext{
* Disciplina de Cirurgia Vascular, Faculdade de Ciências Médicas da Santa Casa de São Paulo, São Paulo, SP.

Não foram declarados conflitos de interesse associados à publicação deste artigo.

Artigo submetido em 23.12.08, aceito em 01.07.09.

J Vasc Bras. 2009;8(3):219-224.

Copyright @ 2009 by Sociedade Brasileira de Angiologia e de Cirurgia Vascular
} 
apresentavam mancha em vinho do porto, varizes e hipertrofia óssea e de tecidos moles chamada originalmente de naevus vasculosus osteohypertrophicus ${ }^{1}$. Sete anos depois, Frederick Parkes Weber descreveu alguns casos com sinais semelhantes aos descritos por Klippel e Trénaunay ${ }^{2}$. Em 1918, Parkes Weber descreveu um caso em que a presença de fístula arteriovenosa estava associada à tríade de sinais referida acima ${ }^{3,4}$.

Inicialmente, as duas síndromes eram estudadas separadamente, e a presença de fístula arteriovenosa era considerada uma característica exclusiva da síndrome de Parkes-Weber. Após a observação de casos nos quais se constatou surgimento de fístulas arteriovenosas não diagnosticadas anteriormente, presença de fístulas capilares em ambas as patologias e semelhanças no quadro clínico e na evolução das doenças, alguns autores começaram a considerá-las como uma única doença, sugerindo, então, para elas, um único nome: síndrome de Klippel-Trénaunay-Weber ${ }^{5}$.

A SKTW é um distúrbio mesodérmico congênito raro de etiologia desconhecida e expressão variável. Pode decorrer de uma mutação para dominante autossômica, quando ocorrida na embriogênese, ou para autossômica dominante com penetrância incompleta. Isso explica a ocorrência familiar. Esta não apresenta um padrão de periodicidade geracional regular, podendo a síndrome ser transmitida por muitas gerações por indivíduos fenotipicamente normais ${ }^{6,7}$.

A SKTW é uma síndrome que produz lesões em diferentes níveis e que apresenta má-formações vasculares diversas, desde capilares, angiodisplasias arteriais ou venosas, má-formações no sistema venoso ou fístulas arteriovenosas. A tríade de sinais descrita acima compreende hemangioma plano, presente em $98-100 \%$ dos pacientes, alterações venosas como má-formações e varizes, presente em $72 \%$ dos casos, e hipertrofia óssea e de tecidos moles, verificada em $67 \%$ das ocorrências da doença. A soma dos três sinais se dá em $63 \%$ dos pacientes, restando uma porcentagem de $37 \%$ com apenas duas característi$\operatorname{cas}^{7,8}$.

A síndrome acomete, na grande maioria das vezes, um único membro inferior (80-85\%). É bilateral nos membros inferiores em $12,5 \%$ dos casos e acomete membros cruzados em 2,5\%. Pode atacar todos os quatros membros, mas em menos de $1 \%{ }^{8}$ das ocorrências.
As manchas em vinho do porto são o primeiro sinal, notado já ao nascimento. Elas podem se localizar em dermátomos, ou apresentar distribuição aleatória. Frequentemente, acometem o membro com hipertrofia, mas também podem afetar outras regiões do corpo e ainda podem variar de profundidade, limitada à epiderme ou acometendo toda a derme ${ }^{9}$.

As má-formações venosas compreendem desde hipoplasia venosa ou ausência de válvulas até aplasia do sistema venoso profundo. São mais comuns em membros inferiores e não é constatada predisposição para o território da safena. Devido às alterações nos sistemas venosos, pode ocorrer flebite, sangramento, trombose venosa profunda, embolia pulmonar e insuficiência venosa crônica ${ }^{4}$.

A presença de angiodisplasias arteriais ou venosas é comum e pode acometer qualquer região do corpo, desde a pele até os órgãos viscerais. Por isso, existe a possibilidade de ocorrer sangramento nesses locais, causando hemoperitônio, hemotórax, hematúria ou enterorragia ${ }^{10}$.

A hipertrofia de ossos e de tecidos moles torna-se evidente nos primeiros anos de vida e progride em diferentes ritmos até os 12 anos. A diferença de comprimento nos membros inferiores varia desde menos de 1 até $12 \mathrm{~cm}$. Assim, esta diferença pode acarretar alterações na marcha e escoliose ${ }^{4}$.

A hipertrofia de tecidos moles parece ser primária e não secundária à má-formação vascular como se acredita$\mathrm{va}^{4}$. A relação do crescimento ósseo com as alterações vasculares também não é bem estabelecida, mas se acredita que o fluxo aumentado de sangue arterial na epífise devido às fístulas arteriovenosas ou capilares pode ser a causa da hipertrofia ${ }^{4}$.

A dor é o sintoma mais frequente e pode ser deflagrada pela doença venosa, pela escoliose gerada pela diferença de membros ou, ainda, por hemangiomas que acometem as mais diversas regiões da superfície corporal, planos profundos ou vísceras.

O duplex scan deve ser o primeiro exame a ser realizado, porque, além de avaliar adequadamente o sistema venoso profundo e superficial, ele é útil na identificação de angiodisplasias e fístulas arteriovenosas. A tomografia computadorizada e a ressonância magnética são complementares ao duplex scan e são usadas com o intuito de 
avaliar as angiodisplasias e determinar suas características para definir o tratamento ${ }^{11,12}$.

Deve ser realizado um escanograma para estudar a diferença de comprimento entre os membros inferiores com vistas a avaliar a necessidade de uso de órteses para compensar a referida diferença entre os membros inferiores.

A arteriografia é útil para identificar e programar a embolização de fístulas arteriovenosas e de angiodisplasias. A realização de uma venografia pode ser útil para avaliar a necessidade de intervenções no sistema venoso, mas essa avaliação deve ser feita com base no exame da venografia em conjunto com o do duplex scan. A angiorressonância vem mostrando resultados promissores em relação à substituição da angiografia ${ }^{8,13,14}$.

O tratamento da SKTW é essencialmente conservador e consiste no uso de compressão e medicação venotônica e linfocinética, bem como de anti-inflamatórios para o controle da dor ${ }^{11,12}$.

O tratamento cirúrgico das fístulas é contraindicado devido aos maus resultados obtidos e à reincidência dos sintomas. A intervenção sobre o sistema venoso superficial é controversa, e deve ser reservada a pacientes muito sintomáticos e com sistema venoso profundo patente. Contudo, alguns autores apresentaram resultados satisfatórios com intervenções sobre o sistema venoso profundo e novas técnicas cirúrgicas para tratamento do sistema venoso superficial como ablação por radiofrequência, endolaser e escleroterapia com polidocanol ${ }^{13-19}$.

O objetivo deste estudo foi avaliar o perfil epidemiológico dos pacientes acompanhados por SKTW no ambulatório de angiodisplasias e linfedema da disciplina de Cirurgia Vascular da Faculdade de Ciências Médicas da Santa Casa de São Paulo.

\section{Métodos}

Foram revisados prontuários de 58 pacientes que estão em acompanhamento no ambulatório de doenças linfáticas e angiodisplasias da disciplina de Cirurgia Vascular da Faculdade de Ciências Médicas da Santa Casa de São Paulo. Os pacientes incluídos na pesquisa tiveram pelo menos uma consulta entre os meses de abril de 2007 e outubro de 2008.

Como não houve alteração do tratamento proposto na ocasião do levantamento, apenas constatação do tipo de tratamento que já estava sendo realizado, a comissão de ética aprovou o trabalho.

Para classificação da doença venosa foi escolhida a classificação clínica CEAP, utilizada de forma cumulativa de acordo com o proposto pela mesma classificação.

\section{Resultados}

Nossos dados mostraram uma distribuição equitativa entre homens e mulheres, havendo 30 homens e 28 mulheres. A idade do início do tratamento variou de 1 a 35 anos, resultando uma média de idade, no momento do diagnóstico, de 12,8 anos. O primeiro sinal notado pela família foi a mancha em vinho do porto, já no nascimento ou no primeiro ano de vida.

Na maioria dos casos, houve acometimento de membro inferior unilateralmente ( $40 \%$ no membro inferior esquerdo e $46 \%$ no membro inferior direito). Em 9\% dos pacientes, a SKTW se apresentou em ambos os membros inferiores, em 3\%, em membro inferior associado a membro superior, e em $2 \%$, apenas no membro superior esquerdo.

Constatamos diferença no comprimento dos membros inferiores em 77,5\% dos pacientes, mas a diferença ultrapassou os $25 \mathrm{~mm}$ em apenas seis pacientes, ou seja, em $10 \%$ dos casos. A diferença variou de 3 a $30 \mathrm{~mm}$, sendo a média de $11,5 \mathrm{~mm}$. Em nenhum destes pacientes houve necessidade de cirurgia ortopédica, sendo indicado apenas o uso de órteses (Figura 1).

O sintoma mais referido foi dor, presente em $68 \%$ dos casos; em $80 \%$ destes pacientes, a dor estava relacionada à estase venosa. Edema foi referido por 31 indivíduos, ou seja, 53\%. A maioria dos pacientes tolerou os sintomas, que foram considerados debilitantes por 10 deles, por nove como causa de dor e por um relacionado à enterorragia de repetição.

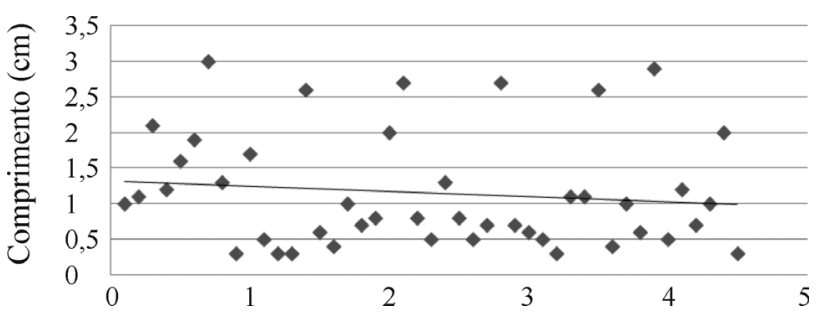

Figura 1 - Diferença de comprimento entre os membros inferiores 
Encontramos 54 pacientes com manchas em vinho do porto, o que representa 93,1\% dos casos. Este foi o primeiro sinal físico observado pela família e também o encontrado com maior frequência. $\mathrm{O}$ segundo sinal mais comum, encontrado em $86,2 \%$ dos casos, foi hipertrofia óssea ou de partes moles.

As alterações venosas estiveram presentes em 72,4\% dos pacientes, manifestas pela presença de varizes em $56,8 \%$, e má-formações vasculares que incluíram angiodisplasias, alterações no sistema venoso profundo e fístulas arteriovenosas em 25,8\%. Além disso, 7,4\% dos indivíduos apresentaram varizes em associação com má-formações.

Quanto à classificação da doença venosa segundo a CEAP, encontramos 56,8\% dos pacientes com varizes visíveis, 53\% com edema e 13,7\% com alterações tróficas secundárias à estase venosa. Três pacientes apresentaram úlcera de estase, apenas um com úlcera ativa e os outros dois com úlceras cicatrizadas (Figura 2).

Foi diagnosticada a presença de fístulas arteriovenosas em quatro pacientes dos 47 estudados, pois 11 pacientes $(8,5 \%$ dos casos) eram muito novos para serem submetidos a exames de imagem. Apenas 6,8\% dos pacientes referiram história familiar. Outras alterações venosas encontradas foram esporádicas.

\section{Discussão}

Devido à observação de que um paciente sem fístulas arteriovenosas no início do quadro pode apresentar fístulas em exames futuros; ao fato de haver fístulas capilares tanto na síndrome de Klippel-Trénaunay quanto na síndrome de Parkes Weber; e à semelhança existente nos sintomas e evolução das duas síndromes referidas, estas são consideradas uma única síndrome denominada síndrome de Klippel-Trénaunay-Weber, concordando com as colocações de Mullins em seu artigo de $1962^{5}$.

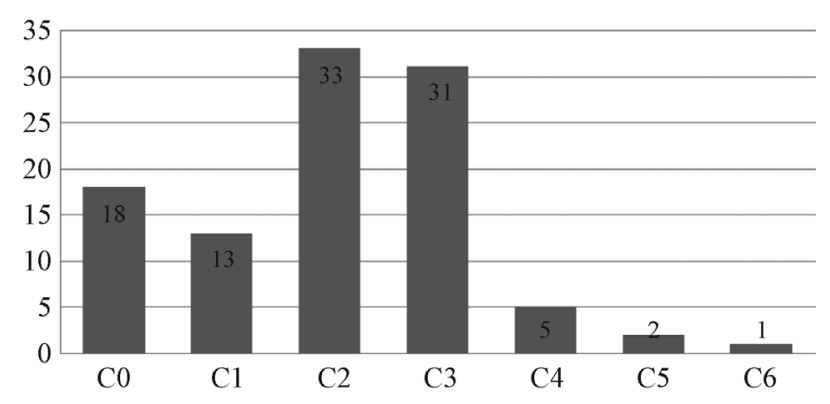

Figura 2 - Classificação CEAP
A distribuição foi equitativa entre homens e mulheres. O diagnóstico é feito na infância e o primeiro sinal é a presença de manchas em vinho do porto, vistas já ao nascimento, sendo também o sinal mais frequente, como foi constatado por Curado, Mulliken e Viljoen ${ }^{9,10,12}$.

Alterações venosas foram verificadas em 72,4\% dos nossos casos; essa mesma distribuição foi encontrada por Curado em 2008 e por Dellis em $2007^{8,11}$. No entanto, enquanto essas alterações venosas são citadas pelos mesmos autores como o segundo sinal mais frequente da síndrome, os nossos dados mostram a hipertrofia óssea e de partes moles presente em $86,2 \%$ dos casos como a segunda alteração mais frequente.

Na classificação CEAP encontramos muitos casos C0 e C1, que são mais freqüentes na infância, sendo que a incidência de alterações tróficas secundárias a estase venosa são raras neste período da vida. No entanto, em geral, as classes C2 e C3 foram as mais encontradas nos nossos casos.

Esse fato parece estar relacionado ao maior número de crianças da casuística, uma vez que nos adultos com mais de 16 anos, encontramos mais casos no estágio C4, C5 e C6. Isso nos faz desenhar a evolução da doença varicosa nos pacientes acometidos pela síndrome e afirmar que o tratamento da doença venosa é importante, dado que o sintoma mais referido e mais debilitante foi a dor relacionada à insuficiência venosa crônica.

A presença de fístulas arteriovenosas foi rara e não houve relação com a gravidade do quadro clínico, nem tampouco com a hipertrofia óssea ou de partes moles. O mesmo padrão foi encontrado por Ziyeh em $2004^{13}$. Esse achado mostra a não existência de uma relação fisiopatológica entre as fístulas arteriovenosas macroscópicas e os demais sinais encontrados na síndrome, como hipertrofia e alterações venosas.

O fator que mais contribuiu para a gravidade da doença, levando em conta os sintomas e os sangramentos, foi a complexidade das má-formações venosas, como sistema venoso profundo avalvulado ou muito hipoplásico e angiodisplasias grandes e em locais de difícil intervenção.

Repercussões clínicas debilitantes são normalmente relacionadas a hipertrofia óssea e de partes moles na infância, a insuficiência venosa crônica nos adultos e a 
apresentações não habituais das alterações venosas que levam a sangramentos de repetição.

O tratamento deve focar o controle clínico da insuficiência venosa crônica e a intervenção em pacientes com varizes calibrosas e sistema profundo patente, além da prevenção de complicações causadas pelas angiodisplasias.

O grande desafio no tratamento desta síndrome é a melhora da qualidade de vida dos pacientes com hipertrofia óssea e de tecidos moles acentuada. O grande avanço no tratamento da SKTW vai ocorrer quando tivermos a possibilidade de diferenciar precocemente os pacientes com quadros mais graves e, a partir daí, desenvolver tratamentos para amenizar ou frear o crescimento ósseo e a hipertrofia de partes moles, seja cirúrgicos ou baseados em terapia para suprimir o efeito da mutação gênica responsável pelas alterações presentes na doença que nos ocupa, permitindo-nos ser mais agressivos no tratamento dos pacientes graves sem causar os efeitos colaterais do tratamento nos pacientes com a forma branda da síndrome.

\section{Conclusão}

A síndrome de Klippel-Trénaunay e a síndrome de Parkes Weber são apresentações diferentes de uma única enfermidade e podem ser estudas em conjunto como síndrome de Klippel-Trénaunay-Weber.

A maioria dos pacientes apresenta os primeiros sinais da doença na infância. Neles, as alterações clínicas de maior morbidade não estão presentes. Este é o momento ideal para reconhecer os portadores da SKTW com o intuito de iniciar medidas terapêuticas que poderão diminuir a morbidade da síndrome.

O tratamento de eleição ainda é conservador e visa o controle da doença venosa e o alívio dos sintomas. O tratamento compressivo deve ser indicado a todos os portadores da doença com vistas a diminuir a evolução da doença venosa periférica, que foi pouco encontrada nas crianças e adolescentes e referida com causa frequente de sintomas nos adultos. O uso de palmilha ou calçado especial ameniza a deformidade na coluna vertebral causada pela diferença nos membros inferiores.

O paciente portador da síndrome deve ser acompanhado em centro de referência com experiência e arsenal terapêutico diversificado para atuar da melhor forma nos casos de insuficiência venosa avançada, fístulas arteriovenosas macroscópicas e angiodisplasias complexas.
A divulgação junto aos colegas pediatras para o diagnóstico precoce da síndrome é essencial no tratamento adequado desta doença.

\section{Referências}

1. Klippel M, Trénaunay P. Du naevus variqueux osteohypertrophique. Arch Gen Med (Paris). 1900;185:641-72.

2. Weber FP. Angioma formation in connection with hypertrophy of limbs and hemi-hypertrophy. Br J Dermatol Syph. 1907:19;231-5.

3. Weber FP. Haemangiectatic hypertrophy of limbs: congenital phlebarteriectasis and so-called congenital varicose veins. Br J Child Dis. 1918;15:13-7.

4. Kihiczak GG, Meine JG, Schwartz RA, Janniger CK. Klippel-Trenaunay syndrome: a multisystem disorder possibly resulting from a pathogenic gene for vascular and tissue overgrowth. Int J Dermatol. 2006;45:883-90.

5. Mullins JF, Naylor D, Redetzki J. The Klippel-TrenaunayWeber syndrome. Arch Dermatol. 1962;86:202-6.

6. Pawel BR, Spencer K, Dormans J. Klippel-Trenaunay syndrome. Arch Dis Child. 2005;90:1127.

7. Wang QK. Update on the molecular genetics of vascular anomalies. Lymphat Res Biol. 2005;3:226-33.

8. Delis KT, Gloviczki P, Wennberg PW, Rooke TW, Driscoll DJ. Hemodynamic impairment, venous segmental disease, and clinical severity scoring in limbs with Klippel-Trenaunay syndrome. J Vasc Surg. 2007;45:561-7.

9. Viljoen D, Saxe N, Pearn J, Beighton P. The cutaneous manifestations of the Klippel-Trenaunay-Weber syndrome. Clin Exp Dermatol. 1986;12:12-7.

10. Mulliken JB, Glowacki J. Hemangiomas and vascular malformations in infants and children: a classification based on endothelial characteristics. Plast Reconstr Surg. 1982;69:412-22.

11. Curado JH, Dutra AK. Angiodisplasias, hemangiomas e máformação vascular: aspectos cirúrgicos. In: Brito CJ. Cirurgia vascular, cirurgia endovascular e angiologia. $2^{\mathrm{a}}$ ed. São Paulo: Revinter; 2008.

12. Curado JH, Campos HGA. Angiodisplasias. In: Maffei FA Doenças vasculares periféricas. $4^{\mathrm{a}}$ ed. Rio de Janeiro: Guanabara Koogan; 2008.

13. Ziyeh S, Spreer J, Rössler J, et al. Parkes Weber or KlippelTrenaunay syndrome? Non-invasive diagnosis with MR projection angiography. Eur Radiol. 2004;14:2025-9.

14. Frasier K, Giangola G, Rosen R, Ginat DT. Endovascular radiofrequency ablation: a novel treatment of venous insufficiency in Klippel-Trenaunay patients. J Vasc Surg. 2008;47:1339-45.

15. Gloviczki P, Driscoll DJ. Klippel-Trenaunay syndrome: current management. Phlebology. 2007;22:291-8.

16. Lee A, Driscoll D, Gloviczki P, Clay R, Shaughnessy W, Stans A. Evaluation and management of pain in patients with Klippel-Trenaunay syndrome: a review. Pediatrics. 2005;115:744-9.

17. Nguyen S, Franklin M, Dudek AZ. Skin ulcers in KlippelTrenaunay syndrome respond to sunitinib. Transl Res. 2008;151:194-6. 
18. Nitecki S, Bass A. Ultrasound-guided foam sclerotherapy in patients with Klippel-Trenaunay syndrome. Isr Med Assoc J. 2007;9:72-5. Comment in: Isr Med Assoc J. 2007;9:112-4.

19. Noel AA, Gloviczki P, Cherry KJ Jr, Rooke TW, Stanson AW, Driscoll DJ. Surgical treatment of venous malformations in Klippel-Trenaunay syndrome. J Vasc Surg. 2000;32:840-7.
Correspondência:

André Luis Costa Villela

Rua General Jardim 595, 14

CEP 01223-011 - São Paulo, SP

Tel./fax: (11) 3258.1706

E-mail: andre.villela@uol.com.br 